\title{
Single Parameter Model for Cosmic Scale Photon Redshift in a Closed Universe
}

\author{
Andre P. Steynberg \\ Andre Steynberg Consulting LLC, Dublin, OH, USA \\ Email: ap.steynberg@gmail.com
}

How to cite this paper: Steynberg, A.P. (2021) Single Parameter Model for Cosmic Scale Photon Redshift in a Closed Universe. Open Journal of Modelling and Simulation, 9, 407-413.

https://doi.org/10.4236/ojmsi.2021.94026

Received: September 10, 2021

Accepted: October 18, 2021

Published: October 21, 2021

Copyright $\odot 2021$ by author(s) and Scientific Research Publishing Inc. This work is licensed under the Creative Commons Attribution International License (CC BY 4.0).

http://creativecommons.org/licenses/by/4.0/ (c) (i) Open Access

\begin{abstract}
A successful single parameter model has been formulated to match the observations of photons from type 1a supernovae which were previously used to corroborate the standard $\Lambda$ cold dark matter model. The new single parameter model extrapolates all the way back to the cosmic background radiation $(\mathrm{CMB})$ without requiring a separate model to describe inflation of the space dimensions after the Big Bang. This single parameter model assumes that spacetime forms a finite symmetrical manifold with positive curvature. For the spacetime manifold to be finite, the time dimension must also have positive curvature. This model was formulated to consider whether the curvature of the time dimension may be related to the curvature of the space dimensions. This possibility is not considered in the more complex models previously used to fit the available redshift data. The geometry for the finite spacetime manifold was selected to be compatible with the Friedmann equation with positive curvature. The manifold shape was motivated by an assumption that there exists a matter hemisphere (when considering time together with a single space dimension) and an antimatter hemisphere to give a symmetrical and spherical overall spacetime manifold. Hence, the space dimension expands from a pole to the equator, at a maximum value for the time dimension. This is analogous to the expansion of a circle of latitude on a globe from a pole to the equator. The three space dimensions are identical so that any arbitrary single space direction may be selected. The initial intention was to modify the assumed geometry for the spacetime manifold to account for the presence of matter. It was surprisingly found that, within the error of the reported measurements, no further modification was necessary to fit the data. The Friedmann equation reduces to the Schwarzschild equation at the equator so this can be used to predict the total amount of mass in the Universe. The resulting prediction is of the order of $10^{51} \mathrm{~kg}$. The corresponding density of matter at the current time is ap-
\end{abstract}


proximately $1.6 \times 10^{-28} \mathrm{~kg} \cdot \mathrm{m}^{-3}$.

\section{Keywords}

Photon Redshift, Cold Dark Matter Model, Single Parameter Model, Cosmic Background Radiation

\section{Introduction}

An interpretation of the Planck Legacy 2018 release (by Di Valentino, Melchiorri and Silk [1]) concluded that spacetime has positive curvature at more than the 99\% confidence level. Positive curvature implies that the spacetime manifold is finite.

Prior to this discovery, the calculation of the expansion of space assumed that spacetime is flat but expanding with time.

When photons are detected from very distant sources, a calculation is necessary which considers the expansion of space with time. As a result, it is necessary to assume the rate of expansion of space in order to calculate the distance travelled by the photon from the source to the detector. The intensity of the detected photon is related to the light path of the photon and it is the light path distance that is measured. This measurement, using photon intensity, must be corrected to account for the density of matter, referred to as cosmic dust, along the light path.

The usual approach to locating the distance for a far-away galaxy assumes that the expansion of space is related to the increase of the wavelength of the photon. A complicated technique is required to calculate a distance travelled through the expanding space dimension. The calculated progression of the space dimensions is then fitted to a modified Friedmann equation into which at least three parameters are added, and the values of these parameters are regressed to get a good fit. The three added parameters are the cosmological constant, $\Lambda$ and the quantities referred to as "dark matter" and "dark energy". This approach was pioneered by Peebles [2] [3].

Gupta, R.P. (2018) [4] published a redshift prediction model based on a hybrid of two effects: recession of distant galaxies due to expansion of the universe, and resistance to light propagation due to cosmic drag. In essence, this introduces an additional model parameter to improve the fit for the standard $\Lambda$ cold dark matter model. It is therefore possible to fit the available data with models which add parameters to the Friedmann equation and then add further models to give a hybrid approach with a better fit.

Another version of a hybrid approach has been used more recently by Fèvre, R. (2021) [5]. Quoting from the introduction, in the first part of this article, we present a closed universe model where expansion begins with a quadratic period linked to a quantum approach to the problem. Compared to models of the un- 
iverse starting with a period of inflation, this model makes it possible to define a simple physical criterion marking the end of the very rapid initial expansion and to give a correct value for the current density of matter. It also follows that the subsequent evolution of the radius of curvature of the universe depends on one parameter: the individual mass of the heaviest particles created at the end of the first phase of expansion.

In the second part, the model studies the evolution of the universe, driven by dark energy, until the hypothetical appearance of a new type of Big Rip, which provides the opportunity to formulate a new hypothesis for the pre-Big Bang universe.

All reasoning is within the framework of the standard Lambda-CDM model.

Nevertheless, a simpler approach has now been found based only on the geometry of a finite spacetime manifold.

\section{Results and Discussion}

The complicated regression approach, used with the standard $\Lambda$ cold dark matter model, can be changed to a single parameter regression approach for a finite and symmetrical spacetime manifold. Rather than using Euclidean geometry, as for the current standard approach, it is now proposed that it is more appropriate to use spherical geometry when the Universe has positive curvature. The calculations are analogous to determining the change in latitude on the surface of the Earth for a known distance over the surface of the Earth and a known ratio of latitude and longitude changes.

Using the assumption that the spacetime manifold has a single radius, $R_{m}$ which is equal for all three space dimensions and for the time dimension (when the space dimensions are measured in light-years and the time dimension is measured in years) leads to a single parameter model to describe the expansion of space with time from the Big Bang event. This expansion of space causes the wavelength of photons to increase in direct proportion with the expansion (when ignoring any potential gravitational redshift or relative motion effects). Spherical geometry is used to locate the time at which the measured photon is emitted.

The space dimension will reach a maximum at time $T$. If the time elapsed from the Big Bang, $t$, is expressed as the ratio $t / T$ then this ratio can be multiplied by $\pi / 2$ to get a change in angle, $\theta$ in radians. This is analogous to a change in latitude except that the proposed convention is to measure the angle from a time zero starting point which is at a position analogous to a pole on a globe. The determination of time zero, at a pole, may be a purely theoretical extrapolation since there is no way to observe what happens prior to the release of the first free photons. The measured redshift corresponding to a photon emitted at time $t_{1}$ and detected at time $t_{2}$ is then determined by the equation:

$$
1+z=\sin \theta_{2} / \sin \theta_{1}
$$

Assuming that our photon detector near Earth is located at about 13.8 billion 
light-years after the Big Bang then the general formula is:

$$
1+z=\frac{\sin \left(\frac{13.8}{T}\right) \pi / 2}{\sin \left(\frac{t}{T}\right) \pi / 2},
$$

where $t$ is the time after the Big Bang time zero when the photon was emitted.

As an illustration, let's assume that cosmic background radiation (CMB) photon was emitted at about 11.01 million years after time zero, then the redshift for this photon will be given by:

$$
1+z=\frac{\sin \left(\frac{13.8}{T}\right) \pi / 2}{\sin \left(\frac{0.01101}{T}\right) \pi / 2}
$$

When $T=24$, then $1+z=1089$ which agrees with our observations. If the estimate for the current age of the Universe or the time at which the first photons are produced is changed then a new value for $T$ can be found to match the observed redshift.

The highest-known redshift radio galaxy (TGSS J1530 +1049 ) [6] is at $1+z=$ 6.72. For this galaxy:

$\sin (13.8 / 24) \pi / 2=0.785389$, so $\sin (t / 24) \pi / 2=0.785389 / 6.72=0.116873$ which gives $t=1.79$ billion years after the Big Bang which is about 12 billion years ago.

So far, the contribution from gravitational redshift has been ignored. This may be reasonable when the spacing between the source and detector is very large. It is assumed, in the following discussion, that the reported cosmological scale factor data has been appropriately interpreted to account for any gravitational redshift effects.

Relative motion for our point of observation has also been ignored. Comprehensive surveys of type 1a supernovae redshift data have recently allowed for the correction of this effect [7].

The light path distance through space for a photon, measured in light-years, is always equivalent to the time elapsed, measured in years, since the emission of the photon. This is known because special relativity works. Localized adjustments, using general relativity, may be necessary where spacetime is distorted by matter.

What has been found is that photons emitted from galaxies which are dated using the standard $\Lambda$ cold dark matter model approach have redshift values which are very similar to the values calculated as described above for a Universe with positive curvature. This is most likely because the additional fitting parameters have transformed the data from the true spherical basis to a flat basis in much the same way that maps are transformed to flat paper representations of the curved surface of the Earth.

Ringermacher and Mead [8] have provided a useful plot of the cosmological scale factor, $a(t)=1 /(1+z)$ as a function of lookback time. 
For $a(t)=0.5$ which corresponds to $1+z=2.0$, they report a value of $t=0.432$ $\times 13.8=5.96$ billion years.

Using this reported value for $t$, the single fitted parameter model prediction for $1+z$ is as follows:

$$
1+z=\frac{\sin \left(\frac{13.8}{24}\right) \pi / 2}{\sin \left(\frac{5.96}{24}\right) \pi / 2}=2.065
$$

Although this is not an exact match, it is well within the data scatter in Figure 2 from Ringermacher and Mead [8].

For a lookback time of $0.6 \times 13.8=8.28$ billion years the calculation gives:

$$
1+z=\frac{\sin \left(\frac{13.8}{24}\right) \pi / 2}{\sin \left(\frac{8.28}{24}\right) \pi / 2}=1.522526 \text {, so } a(t)=0.657 \text { and this is also well within }
$$
the reported data scatter.

In general, for the data range provided by type la supernovae, the fits for the new single fit parameter model and hybrid approaches incorporating the standard $\Lambda$ cold dark matter model are all within the data scatter.

\section{Big Implications}

According to the scientific method, a model fit with fewer parameters is always preferred. So the new single parameter model is an improved representation of reality. This preferred approach does not require the unsubstantiated concepts of "dark energy", or a cosmological constant insertion into the Friedmann equation. Furthermore, this single parameter model extrapolates all the way back to the $\mathrm{CMB}$ radiation without resorting to the use of a separate model which was previously necessary to account for the initial rapid inflation of the space dimension. The success of this model implies that spacetime forms a finite symmetrical manifold. The manifold has a radius, $R_{m}$. At a given point in time, the space dimensions have a radius, $R$.

The Friedmann equation for space with positive curvature is:

$\left(\frac{1}{R} \frac{\mathrm{d} R}{\mathrm{~d} t}\right)^{2}=\frac{8 \pi G}{3} \rho-\frac{c^{2}}{R^{2}}$

At the maximum radius $\left(R_{m}\right)$ the rate of change in $R,(\mathrm{~d} R / \mathrm{d} t)$ is zero so $\frac{8 \pi G}{3} \rho=\frac{c^{2}}{R^{2}}$ and $\rho=M_{u} /(4 / 3) \pi\left(R_{m}\right)^{3}$, where $M_{u}$ is the total mass in the Universe. Rearranging to solve for $M_{u}$ gives: $M_{u}=R_{m} c^{2} / 2 G$.

If $T$ is set to $2.4 \times 10^{10}$ years then $2 \pi R_{m}=(360 / 90) \times 2.4 \times 10^{10}$ so $R_{m}=(4.8 \times$ $\left.10^{10}\right) / \pi$ (in light-years). The calculated mass in the Universe is $9.73 \times 10^{50} \mathrm{~kg}$. For the present time, this translates into a matter density (both dark and visible) of approximately $1.6 \times 10^{-28} \mathrm{~kg} \mathrm{~m}^{-3}$.

No doubt, some refinements to the fitted parameter will be necessary to improve the fit as data are accumulated and reworked with improved corrections 
for gravitational redshift, relative motion, and matter density along the photon pathway.

The model prediction for redshift as a function of past time, with $T=2.4 \times$ $10^{10}$ years, is shown in Figure 1.

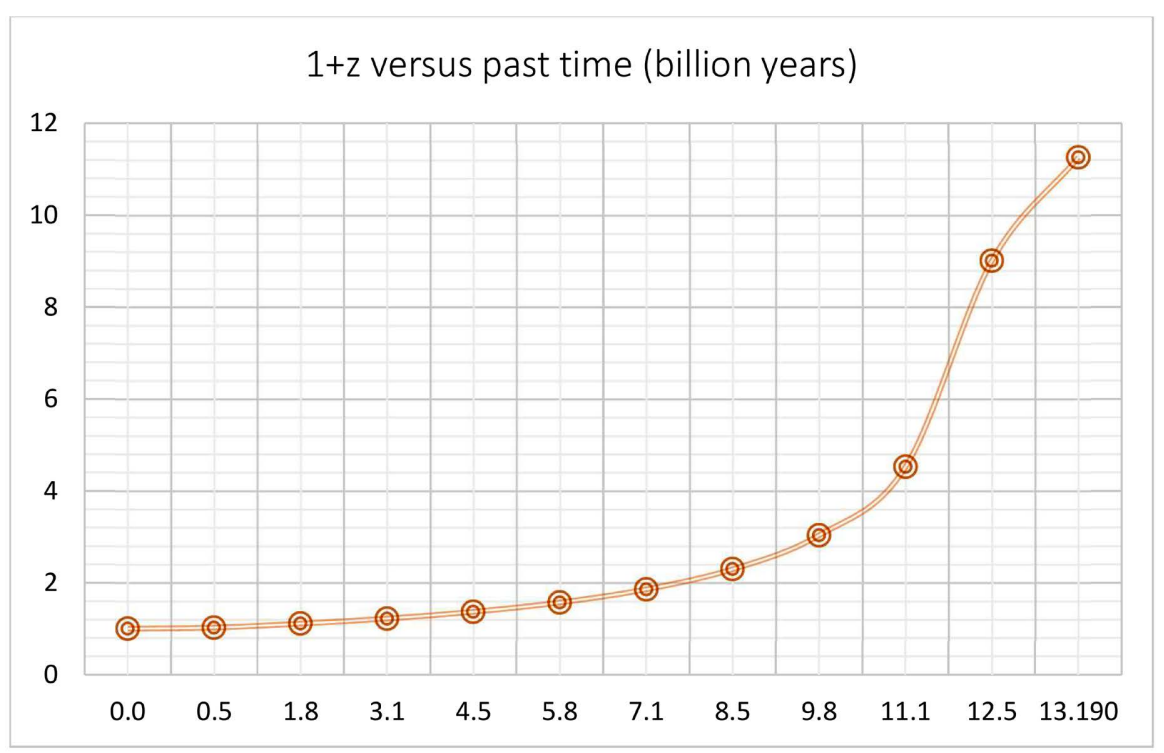

Figure 1. Redshift $(1+\mathrm{z})$ prediction for past time (in billion years), with $\mathrm{T}=24$ billion years.

\section{Conclusion}

Successful redshift predictions, over large time scales (measured in billions of years), have been illustrated using a single parameter model based only on a proposed geometric configuration for a finite spacetime manifold. This implies that the expansion of space with time is the primary explanation for observed photon redshift over large time scales, and the expansion of space is determined by the structure of the spacetime manifold.

\section{Conflicts of Interest}

The author declares no conflicts of interest regarding the publication of this paper.

\section{References}

[1] Di Valentino, E., Melchiorri, A. \& Silk, J. (2020) Planck Evidence for a Closed Universe and a Possible Crisis for Cosmology. Nature Astronomy, 4, 196-203.

https://doi.org/10.1038/s41550-019-0906-9

[2] (2019) Our place in the Universe. Nature Astronomy, 3, 955. https://doi.org/10.1038/s41550-019-0951-4

[3] Peebles, P.J.E. (2019) Cosmology's Early Days. Nature Astronomy, 3, 1055-1057. https://doi.org/10.1038/s41550-019-0960-3

[4] Gupta, R.P. (2018) Mass of the Universe and the Redshift. International Journal of Astronomy and Astrophysics, 8, 68-78. 
https://doi.org/10.4236/ijaa.2018.81005

[5] Fèvre, R. (2021) From the Big Bang to the Big Rip: One Cycle of a Closed Granular Friedmann-Planck Universe, Journal of High Energy Physics, Gravitation and Cosmology, 7, 377-390. https://doi.org/10.4236/jhepgc.2021.72021

[6] Colin, J., Mohayaee, R., Rameez, M. \& Sarkar, S. (2019) Evidence for Anisotropy of Cosmic Acceleration. Astronomy \& Astrophysics, 631, Article No. L13. https://doi.org/10.1051/0004-6361/201936373

[7] Saxena, A., Marinello, M., Overzier, R.A., Rottgering, H.J.A., et al. (2018) Discovery of a Radio Galaxy at $z=5.72$. Monthly Notices of the Royal Astronomical Society, 480, 2733-2742. https://doi.org/10.1093/mnras/sty1996

[8] Ringermacher, H.I. \& Mead L.R. (2014) Model-Independent Plotting of the Cosmological Scale Factor as a Function of Lookback Time. The Astronomical Journal, 148, Article No. 94. https://doi.org/10.1088/0004-6256/148/5/94 